\title{
TITLE:
}

\section{On the Shock Pressure of Surge on a Wall}

$\operatorname{AUTHOR}(\mathrm{S})$ :

NAKAMURA, Shigehisa; TSUCHIYA, Yoshito

\section{CITATION:}

NAKAMURA, Shigehisa ... [et al]. On the Shock Pressure of Surge on a

Wall. Bulletin of the Disaster Prevention Research Institute 1973, 23(3-4): 47-58

\section{ISSUE DATE:}

1973-12

URL:

http://hdl.handle.net/2433/124836

RIGHT: 


\title{
On the Shock Pressure of Surge on a Wall
}

\author{
By Shigehisa Nakamura and Yoshito TsuchiYa
}

(Manuscript received December 22, 1973)

\begin{abstract}
Shock pressure of tsunami bore on a wall-shaped gate is investigated on the basis of an assumption that a surge caused by a dam-breaking can be made to simulate to a tsunami surge in front of the gate in an estuary. Cross-Cumberbatch's theory of shock pressure of surge on a vertical wall is modified and compared with the results of experiment to assure the tip profile of the surge and to estimate the intensity of the maximum shock pressure on the gate.
\end{abstract}

\section{Introduction}

This study treats a problem to estimate exactly the shock pressure of tsunami bore or surge on a wall-shaped gate, in order to establish a criterion in construction of such a gate at a river mouth and a coast. The study is refered to an assumption of similarity between a surge caused by a dam-breaking and a tsumami surge near a river mouth or a beach.

Cross has applied Cumberbatch's theory of shock pressure of a wedge of water mass on a vertical wall to the estimation of the shock pressure of surge impinging on a wall. The authors have rechecked Cross' theory to modify it for the estimation of the shock pressure and considered a problem of reflection of surge in front of a wall, taking into account of an effect of water depth near the wall.

\section{Experiment for Shock Pressure of Surge}

The authors carried out an experiment for measuring the shock pressure of the surge impinging on a wall-shaped gate. Surges were generated by a similar manner which had been used previously by the authors1),2). A schematic figure in Fig. I shows arrangement and experimental conditions used. The wall-shaped gates used in this experiment are vertical and made of acrylite. The two of these gates are composite walls as shown in Fig. 2. Six pressure gauges were installed on the face of the wall-shaped gate, as seen in the figure, in order to find the relation between the characteristics of the surge and the shock pressure. Initial water depths were set $0.5,0.4$ and $0.3 \mathrm{~m}$, which are shown by the notation $h_{0}$ in Fig. 1 . The bed of water tank used for the experiment had been kept horizontal.

An example of shock pressure patterns by a surge on the wall-shaped gate model GA is shown in Fig. 3, in which the first column indicates the number of station where the shock pressure was recorded, the second the elevation of station from the bed in $\mathrm{cm}$, the third the equivalent water depth for the initial shock pressure $p_{p}$, the fourth the saddle pressure $p$, and the fifth the height from the station to the top of the gate.

The experimental condition in the case of Fig. 3(a) is that the surge was intense 
and large enough to overflow the gate. In the case of Fig. 3(b), the surge did not overflow the gate and caused shock pressure at only the lower part of the gate. It may be seen from the figure that most part of the total pressure on the gate is hydrostatic pressure, after the surge reflected and the water began to overflow the gate.

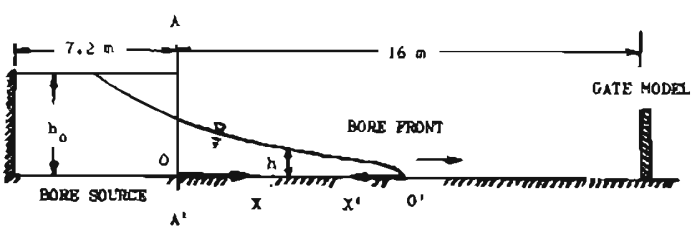

Fig. 1 Schema of experimental arrangement for study on bore
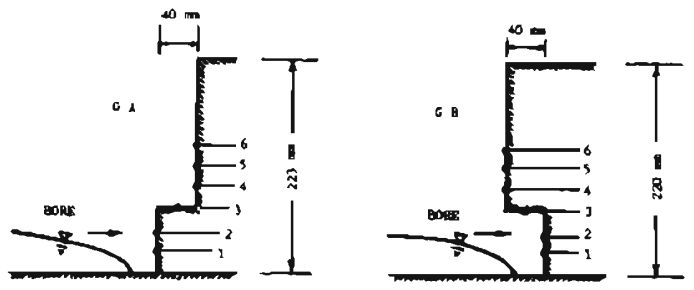

Fig. 2 Schematic plan of water gate models: GA and GB
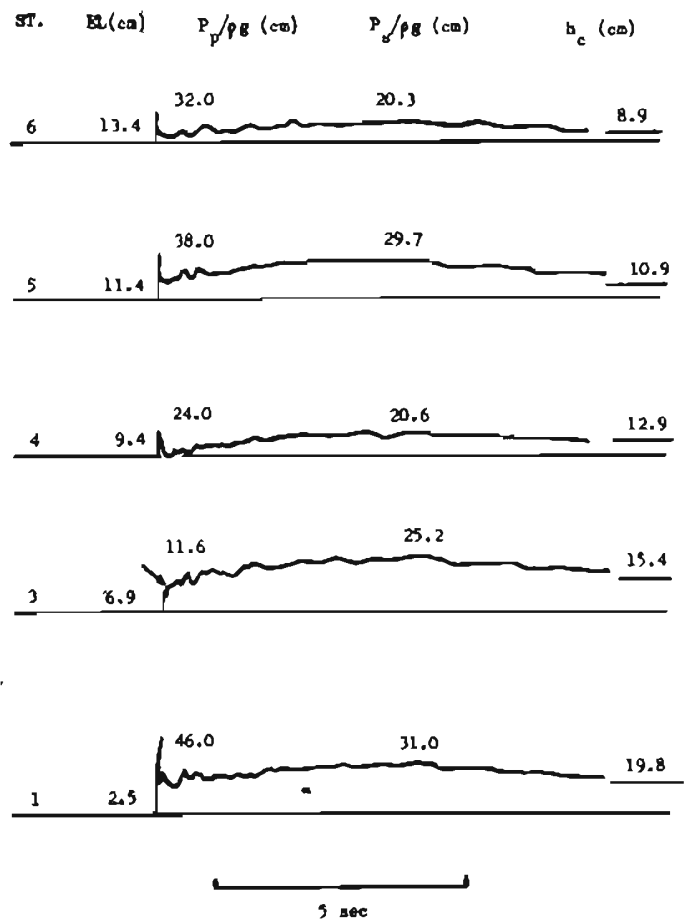

Fig. 3(a) 


$$
\mathbf{s T} \text {. }
$$$$
\mathrm{E}(\mathrm{cm}) \quad \mathrm{P}_{\mathrm{P}} / \mathrm{PB}(\mathrm{cm}) \quad \mathrm{P}_{\mathrm{B}} / \mathrm{gB}(\mathrm{cm}) \quad \mathrm{b}_{\mathrm{c}}(\mathrm{ca})
$$
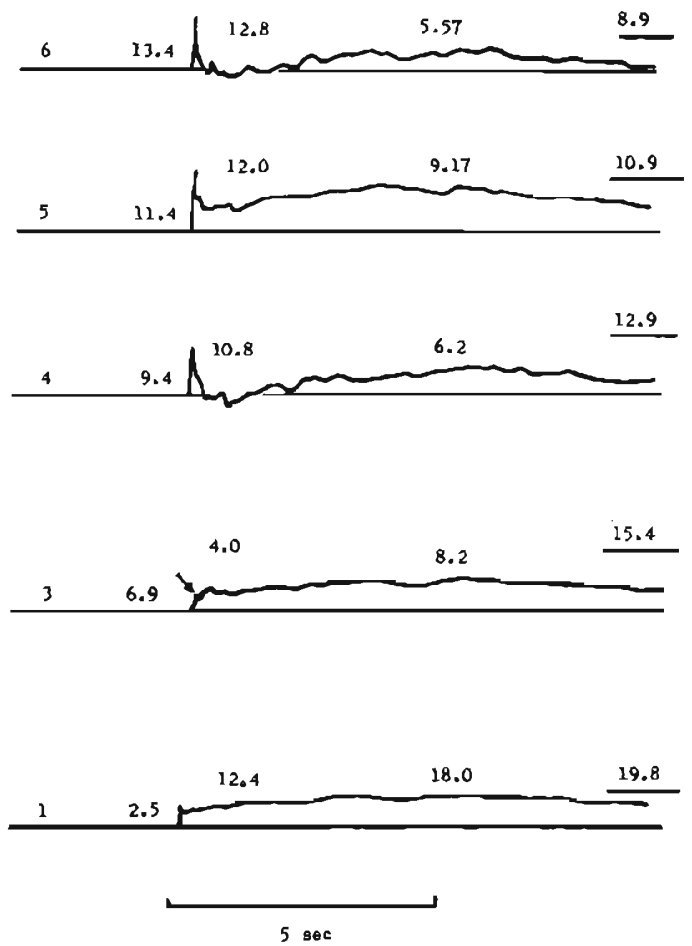

Fig. 3(b)

Fig. 3 An example of records of shock pressure of surge at the wall gate $\mathrm{GA}$ : (a) case of overflowing and(b) case without overflow

As seen in Fig. 3, however, the value of $p_{s} / \rho g$ generally exceeds the head of hydrostatic pressure $h_{c}$.

\section{Profiles of Surge Tip}

There have been many studies of bore or surge as well as run-up of waves on a slope. Some of these studies have been successfully applied by the method of characteristics. In the other studies, Cross, for example, has developed a theoretical approach to determine the tip form of surge on a dry bed. Concerning with the problem of hydraulic bore, various studies have been carried out since Ritter established a theory of dam-breaking function ${ }^{1), 4)}$.

Using a Cartesian co-ordinate as shown in Fig. 1, the solution of dam-breaking function by Ritter can be written as;

$$
u=\frac{2}{3}\left(\frac{x}{t}-\sqrt{g h_{0}}\right), \quad h=\frac{1}{9 g}\left(2 \sqrt{g h_{0}}-\frac{x}{t}\right)^{2}
$$

where $u$ and $h$ are the current velocity and the water depth at the time $t$ and at the 
location $x$ respectively. One of main problems in such a study is to find the tip profile of surge, as shown in Fig. 1, by taking into account of the effect of bottom friction.

The relationship of Eq. (1) is shown in Fig. 4 to compare it with the experimental results of the tip profile. It may be seen from this that the tip of the surge is affected by the effect of bottom friction as well as that of internal turbulence and that the tip of the surge becomes more concave upwards when the surge is smaller.

If there is not any friction on the bed, the velocity at the tip of the surge can be found by Eq. (1) for the given values of $x, l$ and $h_{0}$. When $R=g / C^{2}=0, u / \sqrt{g h_{0}}=2$, where $C$ is Chézy's constant. If the friction on the bed is taken into account, on the other hand, the velocity should be given as a function of the friction factor. In Fig. 5, the relations of Dressler's $s^{5)}$ and Whitham's $s^{6)}$ theory cited by Cross ${ }^{3)}$ are shown by the solid and the chain line respectively for comparison with the results of experiment. It is seen that the tendency of the theoretical curves agrees with the experimental values while the values are much scattered and are larger than the theoretical ones.

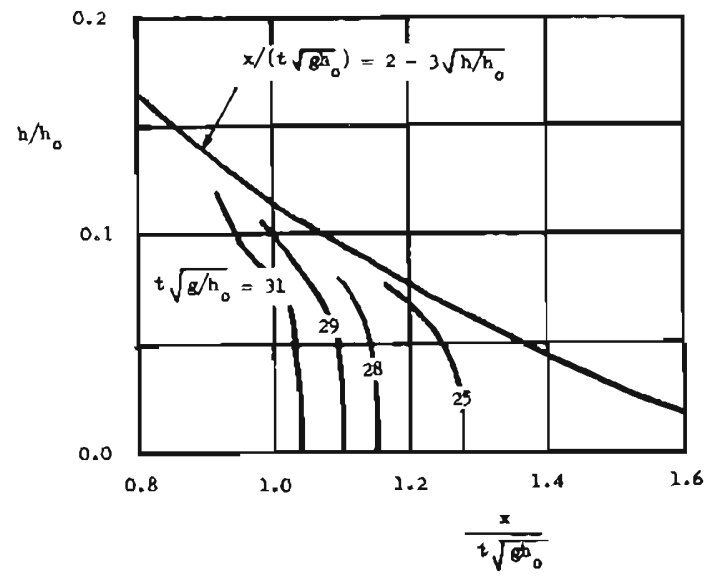

Fig. 4 Ritter's solution and the result of the experiment

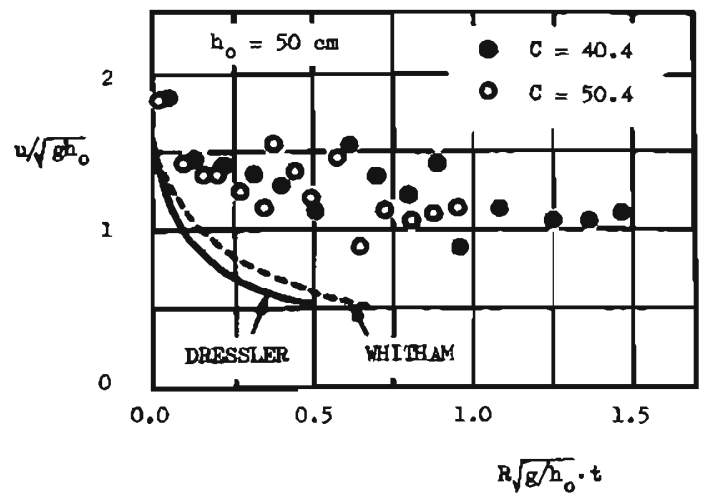

Fig. 5 Velocity of the surge tip 
According to Cross' theory3) based on the assumption that the accerelation of the surge is constant, the profile of the surge can be written as

$$
h=(u / C) \sqrt{2 x^{\prime}},
$$

where $x^{\prime}$ is the distance from the tip of the surge. Using the water depth $h_{0}$ to define the reference velocity of the surge as the form of $u_{0}=\sqrt{g h_{0}}$, the tip profile of the surge in demensionless form can be obtained instead of Eq. (2) as

$$
\left(\frac{h}{h_{0}}\right)=2\left(\frac{x^{\prime}}{h_{0}}\right)\left(\frac{g}{C^{2}}\right)\left(\frac{u}{u_{0}}\right)^{2}=k\left(\frac{x^{\prime}}{h_{0}}\right) .
$$

Eq. (3) is compared with the result of experiment as shown in Fig. 6. The chain lines in the figure are obtained by Cross' theory for $u^{2} / C^{2} h_{0}=6 \times 10^{-3}, 4.5 \times 10^{-3}$, $3 \times 10^{-3}$ and $1.5 \times 10^{-3}$ respectively, which are obtained using the observed values of $u_{0}$ and the estimated value of Chézy's constant. The full lines in Fig. 6 are the result of the experiment for the equivalent parameter of $k=2\left(\mathrm{~g} / \mathrm{C}^{2}\right)\left(u / u_{0}\right)^{2}=5.4 \times$ $10^{-3}, 5.9 \times 10^{-3}$ and $7.1 \times 10^{-3}$ respectively. At the tip of the surge, the result of experiment agrees well with the relationship of Cross' theory, but the theoretical tail form of surge tends to depart from the experimental result. A reason for this may be due to the effect of non-linear surge motion which is neglected in the theory.

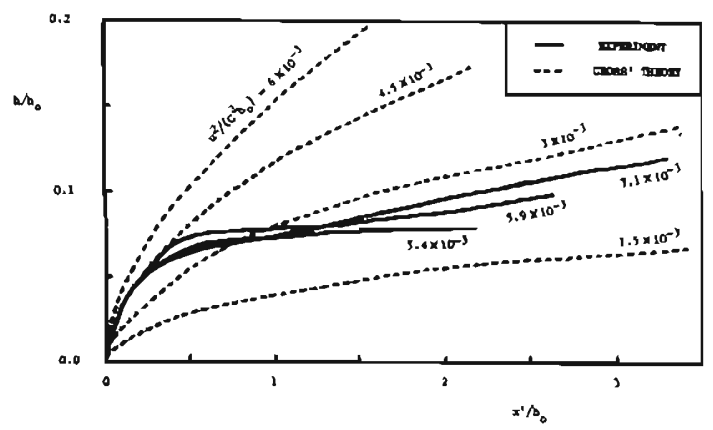

Fig. 6 Profile of the surge tip: Cross' theory and result of experiment

\section{Reflection of Bore at a Vertical Wall}

Now, let us consider a problem of suge or bore running on a flat bed. It is wellknown that the reflection of bore by a wall can be analyzed theoretically by Stoker's theory. By the use of the notations shown in Fig. 8 , the relation between $h_{1} / h_{0}$, $h_{2} / h_{0}$ and $h_{2} / h_{1}$ can be calculated by Stoker's theory, as shown in Fig. 7 . Formerly Ishihara and others ${ }^{7)}$ applied the theory to breakers of periodic waves, which are reflected by a vertical wall, with a good agreement in comparison with their experimental result.

Defining the wave hieght as $H_{1}=h_{1}-h_{0}, H_{2}=h_{2}-h_{0}$ and $H_{21}=h_{2}-h_{1}$, the values can be determined using the water depths of $h_{0}, h_{1}$ and $h_{2}$ in Fig. 7. Numerical examples in the case where $H_{1}=20 \mathrm{~m}$ are tabulated as shown in Fig. 8. This 


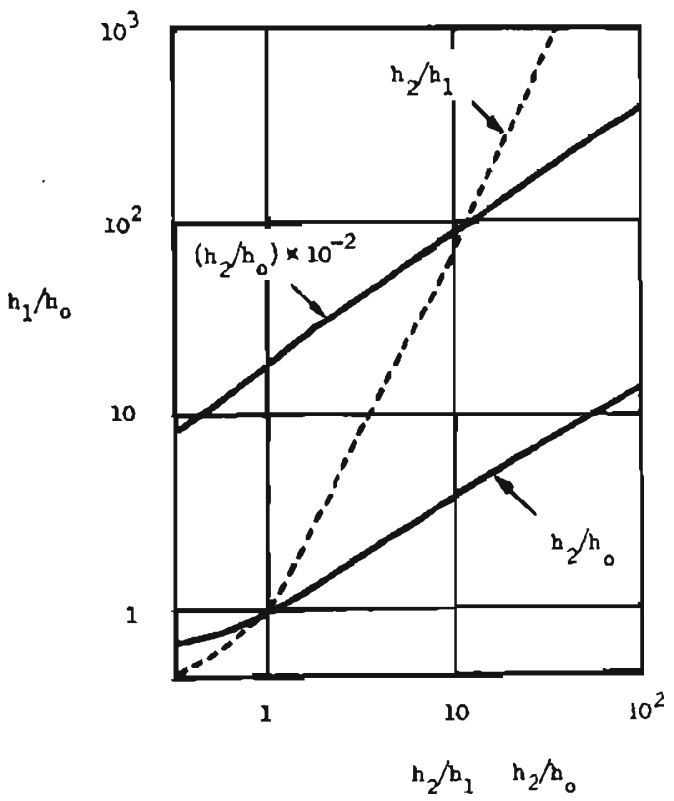

Fig. 7 Reflection of idealized hydraulic bore
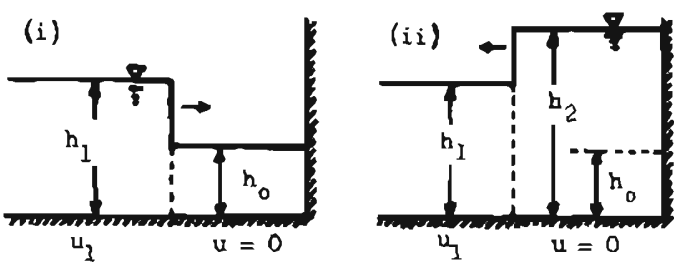

\begin{tabular}{|c|c|c|c|c|c|c|}
\hline & $\mathrm{h}_{\mathrm{o}}$ & $\mathrm{h}_{1}$ & $\mathrm{~h}_{2}$ & $\mathrm{~h}_{1}-\mathrm{h}_{\mathrm{o}}$ & $\mathrm{b}_{2}-\mathrm{h}_{\mathbf{0}}$ & $\mathrm{h}_{2}-\mathrm{h}_{1}$ \\
\hline $\mathrm{a}$ & $\mathrm{l}$ & 21 & 120 & 20 & 119 & 99 \\
\hline $\mathrm{b}$ & 10 & 30 & 65 & 20 & 55 & 35 \\
\hline $\mathrm{c}$ & 20 & 40 & 68 & 20 & 48 & 28 \\
\hline $\mathrm{d}$ & 50 & 70 & 85 & 20 & 35 & 15 \\
\hline
\end{tabular}

Fig. 8 Numerical relations in reflected bore as simulated to tsunami

shows that with the deeper situation of the wall the less the height of waves $\mathrm{H}_{2}$ becomes. If this relation is applicable to such tsunami problems, this suggests that it is preferable to construct the wall as far from the coast as possible in order to reflect perfectly the tsunami bore and to protect the coastal area. Generally the location of the wall should be determined on the basis of various knowledges such as the possibility of practice, topography, geological condition, activity of residents, economical problem and so on. The situation where the coastal area is to be utilized constructing the wall with an opening off the coast is prefered. This leads to the construction of tsunami breakwaters at a bay mouth or off the coast. When there is difficulty in construction 
of breadwaters, the sea wall and wall-shaped gate should be prefered as countermeasures to prevent coastal disasters from tsunami inundation.

\section{Shock Pressure of Surge on Wall-Shaped Gate}

As far as refering to the theory and experiment on hydraulic bores and surges, the pressure acting on a wall can be classified into two parts; the first is the dynamical shock pressure of surge on a structure as seen in Cross' study and the second the hydrodynamic pressure on the structure. It is necessary, of course, to make clear which is active, the shock pressure or the hydrodynamic pressure and to consider the duration of shock pressure in the former case. In this section, a modification of Cross' theory of the shock pressure on a wall is described based on Cross' study.

a) Cumberbatch's theory

Characteristics of shock pressure of surge on a wall-shaped gate are treated refering to Cumberbatch's theory as developed by Cross ${ }^{3}$, though there has been an approach to study the shock pressure of surge by the method of dimensional analysis ${ }^{8}$.

Cumberbatch ${ }^{9}$. developed a theory on the shock pressure of wedge-shaped water mass impinging on a vertical wall, using a schematic model of impinging wedge as shown in Fig. 9. He assumed that the impinging wedge proceeds without change of the form before the tip of the wedge reaches the wall and the effect of gravitational force acting on the wedge is ignored. According to the theory, an approximate expression of the shock pressure by the impinging wedge on the wall can be written as

$$
\frac{P}{\frac{1}{2} \rho u^{2}}=C_{D}\left\{1-\left(\frac{\lambda}{\lambda_{w}}\right)^{2}\right\} ; \quad \lambda=z / u t, \quad C_{p}=C_{p}(\theta),
$$

in which the notation used in the equation is shown in Fig. 9. Pressure distributions in dimensionless form by the approximate solution are drawn in Fig. 10 for the wedge angles of 22.5 and 45 degrees respectively by the chain lines. In the figure, the solid line indicates the pressure distribution by the matched theory. The result of experiment by the authors can be compared with the above approximation theory as shown in Fig. 11. In the comparison, an effort is made to cioncide the time when $t=0$ in the theory with the one when the peak pressure occurs in the experiment. It is seen from the figure that the result of experiment agrees well with the approximate theory. This suggests that a fairly exact evaluation is expected for the peak pressure by an application of Cross-Cumberbatch's theory, when the slope of water

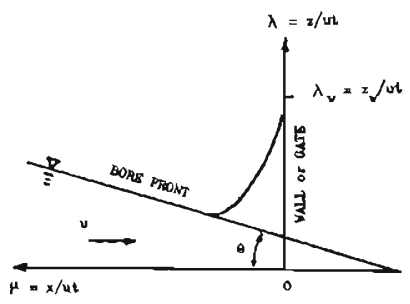

Fig. 9 Cumberbatch's theoretical scheme for shock pressure of surge at a vertical wall 


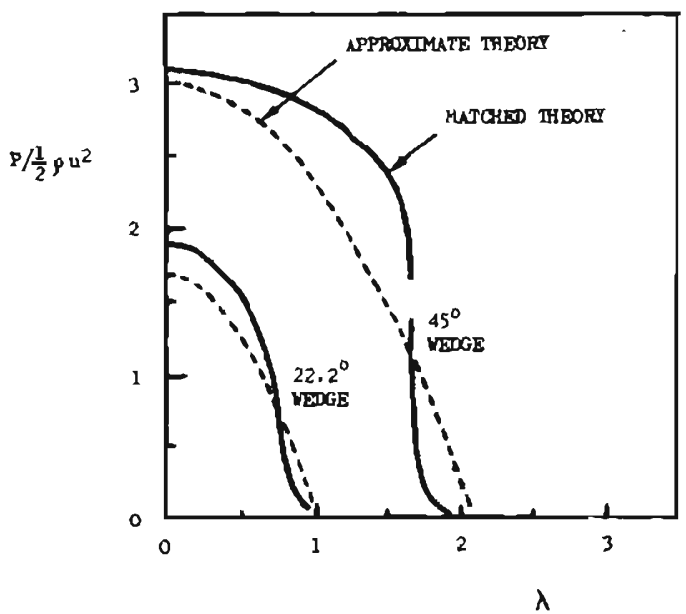

Fig. 10 Distributions of shock pressure on a vertical wall by Cumberbatch's approximation and matched theory

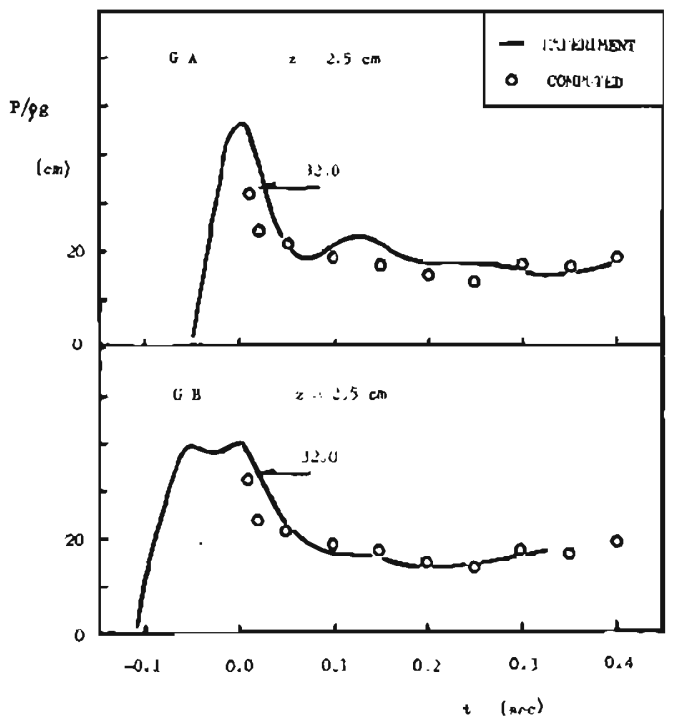

Fig. 11 Cross-Cumberbatch's theory is compared to the experimental nesult for wall-shaped gates GA and $B G$

surface of surge is assumed to correspond to the slope of an equivalent wedge.

b) Peak pressure of surge

Cumberbatch's theory explains that the shock pressure of water wedge can be determined as a function of two variables such as the velocity of the wedge and tip anige of the wedge.

Now refering to $\mathrm{Eq} .(4)$ as an approximate value of shock pressure, the maximum shock pressure occurs at $z=0$ at any time. When an approximation of the surge 
profile is given by $\mathrm{Eq} .(1)$, the slope of the surge tip can be written as

$$
\left(\frac{\partial h}{\partial x}\right)_{0}=-\frac{2}{9}\left(2-\frac{1}{a_{0}}\right)\left(\frac{1}{a_{0}}\right)\left(\frac{h_{0}}{x_{0}}\right) ; \quad\left(\frac{\partial h}{\partial x}\right)_{0}=-\tan \theta
$$

with a definition that $t_{0}=a_{0} x_{0} / \sqrt{g h_{0}}$, where $t_{0}$ is the time that the surge needs to travel the distance $x_{0}$ from the source to the wall-shaped gate.

According to both $\mathrm{Eq}$. (5) and the theoretical result that the coefficient $C_{p}$ in Eq. (4) is a function of the slope of water surface, the maximum shock pressure at $z=0$ is written as

$$
p_{\max }=\frac{1}{2} \rho u^{2} f_{1}\left(h_{0} / x_{0}\right)=-\frac{1}{2} \rho u^{2} f_{2}(\tan \theta) .
$$

The slope of the surge tip which is assumed to be an equivalent water wedge, is expressed by Cross' theory as

$$
\left(\frac{\partial h}{\partial x^{\prime}}\right)_{0}=\frac{1}{\sqrt{2}}\left(\frac{h_{0}}{x^{\prime}}\right)^{-\frac{1}{2}}\left(\frac{1}{a_{0}^{-}}\right)\left(\frac{g}{C^{2}}\right)^{-\frac{1}{2}} .
$$

A practice to determine the value of $\tan \theta$ is to observe the tip profile of surge near the wall-shaped gate as an equivalent water wedge and to estimate the values that $h_{0} / x^{\prime}=3$ to $5, a_{0}=0.6$ to 0.8 and $g / C^{2}=160$. The theoretical relationship by Cumberbatch gives 1.1 to 1.2 for the value of $C_{p}$.

The maximum shock pressure of the surge is affected intensively by the slope of the water surface as mentioned above. The relation between $C_{p}$ and $\tan \theta$ by equating approximately the value of $\tan \theta$ to $\left(\partial h / \partial x^{\prime}\right)_{0}$ can be shown in Fig. 12 . The theoretical curve is given for the condition that $a_{0}=t \sqrt{g h_{0}} / x_{0} \sim 1$ as shown in Fig. 12 where the dots and circles indicate the results of experiment. The values

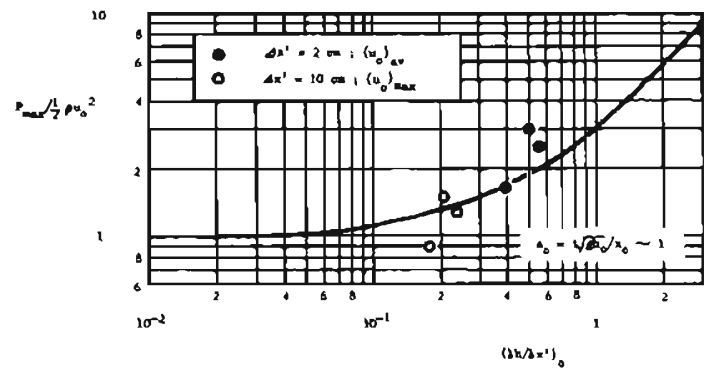

Fig. 12 Profile of the surge tip and the peak value of shock pressure of the surge at wall gate

shown by dots were obtained from the slope at the distance $2 \mathrm{~cm}$ from the concave surge tip and the averaged velocity of the surge tip, and the circles also from the slope at the distance of $10 \mathrm{~cm}$ from the sharp surge tip and the maximum velocity of the surge tip.

c) Hydrostatic pressure of reflected surge on wall-shaped gate

As is found in Fig. 7, the value of $h_{1} / h_{0}$ determines the water depth of crest height 
above the bed $h_{2}$. When the value of $h_{2}$ takes a very large one, as in the case of (a) of the figure, the hydrostatic pressure for the water depth of $h_{2}$ should be taken into account of estimating the maximum pressure on the gate as well as the dynamic shock pressure.

Exactly speaking, additional conditions should be given for the problem of shock pressure caused by periodic waves or tsunamis. An approximate evaluation will give whether the maximum pressure on the wall-shaped gate mainly consists of the shock pressure of the impinging surge or the hydrostatic pressure of the reflected surge. Variations in the shock and the hydrostatic pressure are shown in Fig. 13 for comparison with the theoretical ctrves for the three gate models described already. In the figure, the solid lines indicate the maximum shock pressure on the gate computed refering to Cross-Cumberbatch's theory, the dots are the maximum shock pressure estimated using the values of the approximate total force of surge on the gate which are calculated by the use of Eq. (4) and the value of $z_{20}=h_{w}(l)$ determined from Cumberbatch's theory, and the circles are determined by the same manner as that for the dots, but the value of $h_{p}$ is used instead of $h_{w}$. In this case the values of $h_{p}$ and the maximum height of the water surface at the gate were estimated from the frames of $16 \mathrm{~mm}$ cine-photographs recorded.

In the modification of Cross-Cumberbauch's thory described already, several assumptions are introduced; 1) the surge front keeps a constant form at any time,

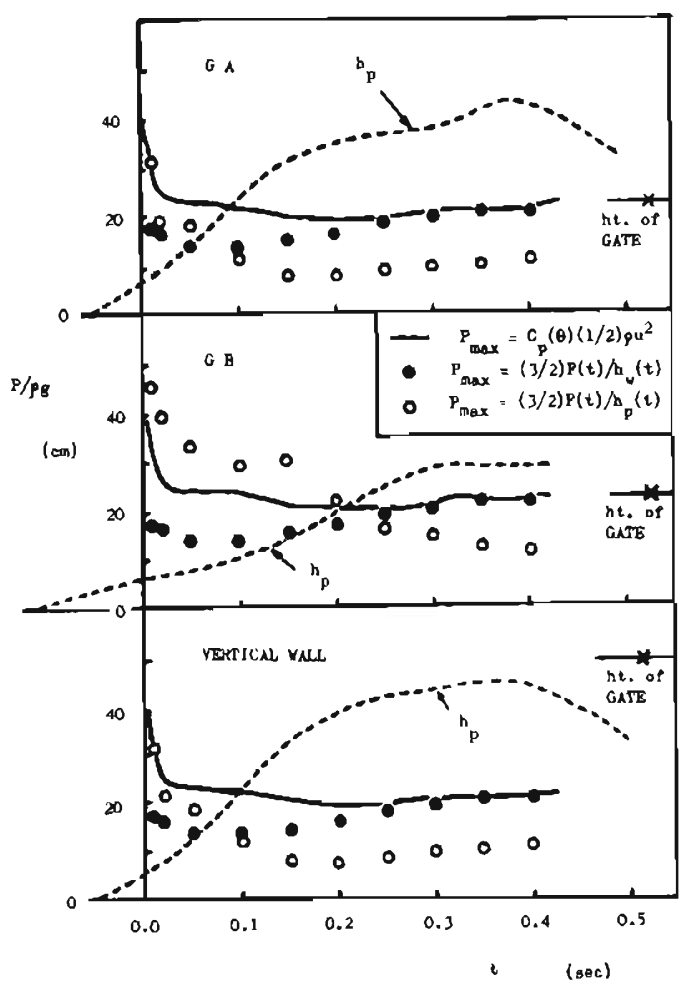

Fig. 13 Maximum pressure of surge at wall-shaped gates 
2) the velocity of surge is constant and 3) the slope of water surface of the surge can be determined as that of the water surface at the distance where $x^{\prime}=u t$, and the value of angle of the surge is obtained from the slope of the water surface to fit the experimental result to Cross-Cumberbatch's theory.

It can be expected that the experimental results agree well with the theoretical ones within the initial range of less than the period of $0.1 \mathrm{sec}$. While the period of 0.1 sec after impinging of surge to the gate, the gravitational force acting on the surge seems to be negligible as in Cumberbatch's theory. Since the gravity effect makes the other motion of water mass after the time elapse of about $0.1 \mathrm{sec}$, the value of $h_{p}$ generally exceeds that of $p_{\mathrm{max}} / \rho g$. When the value of $h_{p}$ exceeds the crown height of gate, the running water begins to overflow the gate. These phenomena were also found in the records shown in Fig. 3(a). It is remarkable in Fig. 13 that the hydrostatic pressure on the vertical wall is larger than that in the cases of the wall-shaped gates of types of GA and GB. For the case where there is no expectation to overflow the gate, the record is shown in Fig. 3(b), in which the value of $p_{p} / \rho g$ may be less important than the values of $p_{s} / \rho g$ and $h_{c}$.

Additional remarks should be given on the duration characteristics of the shock pressure to decide whether the surge makes destructive action against the wall-shaped gate or not, although the peak pressure of the surge is the most important factor. Based on the numerical results shown in Fig. 11, the expected peak pressure on the wall is nearly equal to the shock pressure at the time when $t=0.1 \mathrm{sec}$. This suggests that the destructive action of the surge may occur within the duration of shock pressure.

\section{Conculsions}

The authors have investigated the method of estimation of the shock pressure of tsunami on a wall-shaped gate under the assumption that the surge on a flat bed can be simulated to tsunami bore in an estuary. The conclusive remarks in this paper can be summarized as;

1) Dynamical characteristics of the surge tip can be estimated by an application of Cross' theory.

2) The peak shock pressure of the surge on a wall-shaped gate can be estimated by Cumberbatch's theory as was done by Cross. For practical purposes, a modification of Cross-Cumberbatch's theory of shock pressure of surge is proposed on the basis of considerations of parabola profile of surge tip. It was concluded that the estimation by the modified theory agrees well with the results of experiment of the variation in the shock pressure of the surge on a wall-shaped gate.

3) Hydrostatic pressure of reflected surge on the wall-shaped gate is generally an important factor as well as the peak shock pressure of the surge on it.

A complete method to estimate the maximum shock pressure of tsunami bore or surge on a wall should be established for practice, refering to the additional results of experiment and the theoretical considerations.

\section{Acknowledgements}

The authors wish to express their heartfelt acknowledgements to Mr. T. Yoshitomi, 
Hokoku Industry Co., for his kind support in conducting this investigation. Thanks are due to $\mathrm{Mr}$. T. Yasuda, post graduate student of Kyoto University, for his kind assistance in the experiment.

\section{References}

1) Nakagawa, H., S. Nakamura and Y. Ichihashi: Generation and developement of a hydraulic bore due to the breaking of a dam (1), Bull. Disaster Prevention Research Institute, Kyoto University, Vol. 19, Pt. 2, No. 154, 1969, pp. 1-17.

2) Disaster Prevention Research Association: A study on shock pressure of tsunami (first report), 1973, pp. 1-39.

3) Cross, R.H.: Water surge forces on coastal structures, Technical Report HEL-9-10, University of California, Berkeley, 1966, pp. 1-106.

4) Stoker, J. J.: Water waves, Interscience Pub., N. Y., 1957, P. 312.

5) Dressler, R. F.: Hydraulic resistance effects upon the dam-breaking functions, Jour. Res. US National Bureau of Standards, Vol. 49, 1952, pp. 217-225.

6) Whitham, G. B.: The effect of hydraulic resistance on the dam-break problem, Proc. Royal Society, Ser. A, Vol. 227, 1955, pp. 399-407.

7) Ishihara, T., Y. Iwagaki and Y. Suzuki: On design of sea dike, especially on its effective height, Proc. Coastal Engineering in Japan 1955 pp. 57-70.

8) Fukui, Y., N. Nakamura, H. Shiraishi and H. Sasaki: Hydraulic study on tsunami, Coastal Engineering in Japan, JSCE, Vol. 6, 1963, pp. 67-82.

9) Cumberbatch, E.: The impact of a water wedge on a wall, Jour. Fluid Mech., Vol. 7, 1960, pp. 353-373. 